\title{
Organizational refinements of Nash equilibrium
}

\author{
Takashi Kamihigashi $^{1} \cdot$ Kerim Keskin $^{2} \cdot$ Çağrı Sağlam ${ }^{3}$
}

Accepted: 20 March 2021 / Published online: 23 April 2021

(C) The Author(s), under exclusive licence to Springer Science+Business Media, LLC, part of Springer Nature 2021

\begin{abstract}
Strong Nash equilibrium (see Aumann, 1959) and coalition-proof Nash equilibrium (see Bernheim et al., 1987) rely on the idea that players are allowed to form coalitions and make joint deviations. Both of these notions consider cases in which any coalition can be formed. Accordingly, there may arise "conflicts of interest" that prevent a player from choosing an action that simultaneously meets the requirements of two coalitions to which he or she belongs. Here, we address this observation by studying an organizational framework such that the coalitional structure is (i) motivated by real-life examples where players cannot form some coalitions and (ii) formulated in such a way that no conflicts of interest remain. We define an organization as an ordered collection of partitions of the player set such that any partition is coarser than the partitions that precede it. For any given organization, we introduce the notion of organizational Nash equilibrium. We analyze the existence of equilibrium in a subclass of games with strategic complementarities and illustrate how the proposed notion refines the set of Nash equilibria in some examples of normal form games.
\end{abstract}

Keywords Nash equilibrium · Refinements · Coalitional structure · Organizational structure $\cdot$ Games with strategic complementarities

JEL Codes C72

Kerim Keskin

kkeskin@ada.edu.az

1 Kobe University, Kobe, Japan

2 ADA University, Baku, Azerbaijan

3 Bilkent University, Ankara, Turkey 


\section{Introduction}

The notion of Nash equilibrium is the most prominent solution concept in gametheoretic analysis (see Nash 1951). Motivated by the fact that having multiple equilibria might be undesirable, numerous papers in non-cooperative game theory focus on approaches for refining the set of Nash equilibria (see Aumann 1959; Selten 1965, 1975; Myerson 1978; Kohlberg and Mertens 1986; Bernheim et al. 1987, among others). These equilibrium refinements utilize different methods to decrease the number of equilibria; for example, there are refinements that allow players to form coalitions and make joint deviations. Among such coalitional refinements, we are mainly concerned with strong Nash equilibrium (SNE) (see Aumann 1959) and coalition-proof Nash equilibrium (CPNE) (see Bernheim et al. 1987 ) in this paper. ${ }^{1}$ To elaborate on these notions, one can say that the members of any particular coalition would prefer not to deviate collectively from a SNE strategy profile. As coalitions do not face too many restrictions in choosing their joint deviations, the set of SNE generally turns out to be empty. Expanding from this observation, Bernheim et al. (1987) propose the notion of CPNE according to which coalition members cannot make binding commitments to deviate (i.e., the deviation agreements must be "self-enforcing"2). Accordingly, a strategy profile is said to be coalition-proof, if no coalition is able to deviate from that profile via self-enforcing agreements.

An important observation would be that both of these refinements consider cases in which any coalition can be formed. Yet in real-life situations, we see many instances where some coalitions cannot be formed. Moreover, even when a particular coalition is formed, this does not necessarily imply that all of its subcoalitions would be formed. Indeed, a game might have players that hate/dislike each other or who simply cannot communicate to participate in a coalition. Consider, for example, two countries with a history of bad relations. These countries might not prefer to create a two-player coalition; or even if they meet at a global association, they might still refuse to form the two-player subcoalition. ${ }^{3}$ Following the studies on "conference structures" in the vein of Myerson (1980), consider also two academic scholars at a conference, neither of whom has met the other or any colleague who could have brought them together. Even if they belong to the same society, these two scholars cannot collaborate. We can also provide examples in which some coalitions cannot be formed because of some institutional rules or

\footnotetext{
1 These are known to be the most prominent coalitional refinements of Nash equilibrium. For studies on these refinements, see Bernheim and Whinston (1987), Greenberg (1989), Dutta and Sen (1991), Konishi et al. (1997a), Konishi et al. (1997b), Konishi et al. (1999) among others. In addition to these equilibrium notions, one can find other refinements of Nash equilibrium also utilizing certain types of coalitional stability: strong Berge equilibrium (Berge 1957), the largest consistent set (Chwe 1994), negotiationproof Nash equilibrium (Xue 2000), etc.

2 A deviation is self-enforcing if none of the subcoalitions of the deviating coalition desires a further deviation.

3 One might argue that the countries would form a two-player coalition if it benefited them, but (i) forming a coalition does not necessarily make them better off at the equilibrium, given that there is strategic interaction between the players; and (ii) participating in the same coalition could bring some indirect costs which cannot be implemented into the payoff functions of the game.
} 
regulations. For example, the competition laws in many countries prohibit cooperation between firms that compete in the same market. However, firms are free to cooperate with any other firm with whom they do not compete. Along a similar line, in sport competitions, a player in a team is forbidden to form a coalition with a player of the opponent team while he or she freely cooperates with his or her teammates.

Another important observation on SNE and CPNE lies within the actions of the players. SNE allows a coalition to choose a joint strategy profile, or a deviation, that may be inconsistent with the rational choices of its subcoalitions. In other words, even if a coalition wishes to choose a Pareto deviation, one of its subcoalitions may not be willing to follow that deviation, which may not be Pareto optimal given the actions of the non-members. In such a case, some of the members of that subcoalition face what we call a "vertical conflict of interest" (one coalition tells you to do something, but another tells you to do something else). CPNE actually overcomes this conflict by restricting each coalition to respect the rationality of all of its subcoalitions. Be that as it may, CPNE still allows a coalition to choose a joint strategy profile that may be inconsistent with the rationality of another coalition that is neither a subcoalition nor a supercoalition, but that shares some players. In such a case, some of the common members of those coalitions face what we call a "horizontal conflict of interest" (once again, one coalition tells you to do something, but another tells you to do something else).

With regard to the former observation, it seems worthwhile to consider an equilibrium refinement that does not require the formation of all possible coalitions. With regard to the latter observation, there may be a restricted set of coalitions that contains neither vertical nor horizontal conflicts of interests. In this paper, we seek to address these concerns by formulating a new equilibrium refinement. The refinement that we propose (i) does not suffer from the problems embedded in vertical and horizontal conflicts of interests; and (ii) can be a useful alternative to the notions of SNE and CPNE, and even to the notion of Nash equilibrium, in cases where some coalitions are active (i.e., formed) and the others are not.

Note also that the former observation calls for a general coalitional structure that does not necessarily include some coalitions, whereas the latter observation calls for a specific framework that restricts the set of coalitional structures to be studied. More precisely, to eliminate vertical conflicts of interest, every coalition should respect the rationality of its members and subcoalitions (as in the case of CPNE). In addition to this, to avoid horizontal conflicts of interest, the coalitional structure should be formulated in such a way that for any pair of active coalitions, either the coalitions are disjoint or one coalition contains the other. This is referred to as an organizational structure.

Intuitively, in a non-cooperative game, players may form coalitions if they are allowed to. We are particularly interested in situations where if a player is a member of a coalition, then he or she cannot be a member of another coalition. Accordingly, the set of all active coalitions turns out to be a partition of the player set. As coalitions may unite to form greater coalitions, in the next step, we have another partition of the player set, which is coarser than the former partition. This recursively leads to a collection of partitions such that each partition is coarser than 
the partitions that precede it. We call it an organization. For an example, consider a university as a set of faculty members such that each faculty member belongs to one department and each department belongs to one school. Another example is a football team with footballers and their positions (e.g., defenders, midfielders, and strikers); or a company with employees, units, departments, and divisions.

In this paper, given any organizational structure, we define the notion of organizational Nash equilibrium (ONE), ${ }^{4}$ for which we utilize strict Pareto dominance to describe the coalitions' preferences (Sect. 2.3). ${ }^{5}$ We then study some examples of normal form games through which we can understand how our organizational refinement works and how its predictions are different from those made by SNE/CPNE (Sect. 2.4). We analyze the existence of equilibrium for a subclass of games with strategic complementarities (Sect. 3.1). We also provide a monotonicity property indicating that as one considers "greater" organizations, the equilibrium set is more refined (Sect. 3.2). We conclude in Sect. 4.

\section{Organizational refinements of Nash equilibrium}

\subsection{Preliminaries}

Let $\Gamma=\left(N,\left(X_{i}\right)_{i \in N},\left(u_{i}\right)_{i \in N}\right)$ be an $|N|$-player normal form game in which $N$ denotes the finite set of players, $X_{i}$ denotes the strategy set for player $i \in N$, and $u_{i}$ : $\prod_{i \in N} X_{i} \rightarrow \mathbb{R}$ denotes the utility function for player $i \in N$. For any coalition $S \subset N$, let $X_{S} \equiv \prod_{i \in S} X_{i}$ denote the set of strategy profiles for the members of this coalition; and further, set $X_{-S}=X_{N \backslash S}$ and $X_{N}=X$.

First, we define a Nash equilibrium.

Definition 2.1 Given a normal form game $\Gamma$, a strategy profile $x^{*} \in X$ is a Nash equilibrium if for every $i \in N$ and every $x_{i}^{\prime} \in X_{i}: u_{i}\left(x^{*}\right) \geq u_{i}\left(x_{i}^{\prime}, x_{-i}^{*}\right)$.

Being one of the most prominent coalitional refinements in the literature, the notion of strong Nash equilibrium (SNE) is defined as follows.

Definition 2.2 Given a normal form game $\Gamma$, a strategy profile $x^{*} \in X$ is a strong Nash equilibrium (SNE) if for no coalition $S \subset N$, there exists some $x_{S}^{\prime} \in X_{S}$ such that for every $i \in S: u_{i}\left(x_{S}^{\prime}, x_{-S}^{*}\right)>u_{i}\left(x^{*}\right)$.

\footnotetext{
${ }^{4}$ Our approach may seem rather ad hoc. However, we should note that we have no intention of imposing a certain type of coalitional structure. We simply argue that (i) there are many real-life examples in which some coalitions/subcoalitions are inactive and (ii) organizations make up an important subset of such coalitional structures.

5 Two related strands of literature are the analysis of permissible coalitions in $n$-person cooperative games (see Maschler 1963; Myerson 1980; Calvo and Gutiérrez-López 2015, among others) and the analysis of coalition formation games (see Ray and Vohra 1999; Pápai 2004; Kimya 2020, among others), in the sense that all these studies consider cases where some coalitions are not formed as well. To our understanding, however, there is not a strong relation or resemblance between our results and their results, since they are not particularly interested in organizational structures or equilibrium refinements in noncooperative games.
} 
Bernheim et al. (1987) introduce "self-enforceability" in order to weaken the coalitional stability requirement. Accordingly, the authors define coalition-proof Nash equilibrium (CPNE). Now, before proceeding to the definition of CPNE, we define a reduced game.

Definition 2.3 Given a normal form game $\Gamma$, a coalition $S \subset N$, and a strategy profile $x_{-S} \in X_{-S}$, the reduced game $\left.\Gamma_{S}\right|_{x_{-S}}=\left(S,\left(X_{i}\right)_{i \in S},\left(v_{i}\right)_{i \in S}\right)$ is defined in such a way that for every $i \in S, v_{i}: X_{S} \rightarrow \mathbb{R}$ is given by $v_{i}\left(x_{S}^{\prime}\right)=u_{i}\left(x_{S}^{\prime}, x_{-S}\right)$.

The following is the definition of CPNE.

Definition 2.4 Given a normal form game $\Gamma$,

(i) If $\Gamma$ is a single-player game, then a strategy profile $x^{*} \in X$ is a coalition-proof Nash equilibrium (CPNE) if and only if $x^{*}$ maximizes $u_{1}$.

(ii) Let $|N|>1$ and assume that the set of CPNE is defined for any game with less than $|N|$ players. Define a strategy profile $x^{*} \in X$ to be self-enforcing if for every $S \subsetneq N: x_{S}^{*} \in \operatorname{CPNE}\left(\left.\Gamma_{S}\right|_{x_{-S}^{*}}\right)$. Then a strategy profile $x^{*} \in X$ is a $C P N E$ if and only if it is self-enforcing and there is no other self-enforcing strategy profile $x \in X$ such that for every $i \in N: u_{i}(x)>u_{i}\left(x^{*}\right)$.

In the following, we first introduce a notation relevant to our definition of organizational refinements. In the meantime, we also provide equivalent definitions for the notions of SNE and CPNE, which are consistent with the introduced notation and apparently comparable to the notion of organizational Nash equilibrium (ONE).

\subsection{Alternative characterizations of SNE and CPNE}

For any strategy profile $x \in X$ and any set of strategy profiles $Y \subset X$, define $B_{N}(x, Y)$ or $B_{N}(Y)$ as follows:

$$
B_{N}(x, Y)=B_{N}(Y)=\left\{y \in Y \mid \nexists z \in Y, \forall i \in N: u_{i}(y)<u_{i}(z)\right\} .
$$

For any coalition $S \subset N$ with $|S|<|N|$, any strategy profile $x \in X$, and any set $Y_{S} \subset X_{S}$, define ${ }^{6}$

$$
B_{S}\left(x, Y_{S}\right)=\left\{y \in X \mid y_{S} \in Y_{S} \text { and } \nexists z_{S} \in Y_{S}, \forall i \in S: u_{i}\left(y_{S}, x_{-S}\right)<u_{i}\left(z_{S}, x_{-S}\right)\right\} .
$$

The projection of $B_{S}(x)=B_{S}\left(x, X_{S}\right)$ onto $X_{S}$ is the set of "rational" (or weakly Pareto optimal) responses of coalition $S$ to the strategy profile $x \in X$. This means that if $y \in B_{S}(x)$, then $y_{S}$ is a rational response of coalition $S$ to the other players' joint strategy profile $x_{-S}$. Finally, for any strategy profile $x \in X$, define

$$
B(x)=\bigcap_{S \subset N} B_{S}(x) .
$$

A fixed point ${ }^{7}$ of this correspondence $B$ turns out to be a SNE.

\footnotetext{
${ }^{6}$ Note that $B_{S}\left(x, Y_{S}\right)$ does not impose any restriction on the strategy profile for the non-members of coalition $S$; i.e., if $y \in B_{S}\left(x, Y_{S}\right)$, then for any $x_{-S}^{\prime} \in X_{-S}:\left(y_{S}, x_{-S}^{\prime}\right) \in B_{S}\left(x, Y_{S}\right)$.

7 A fixed point $x$ of a correspondence $F: X \rightarrow X$ is defined to satisfy $x \in F(x)$.
} 
Proposition 2.1 A strategy profile $x \in X$ is a strong Nash equilibrium if and only if $x \in B(x)$.

Proof See the Appendix.

Unfortunately, without certain restrictions on deviations, the correspondence above can easily be empty-valued. This is because what we call a "vertical conflict of interest" arises.

Definition 2.5 Given a strategy profile $x \in X$, two coalitions $S, S^{\prime} \subset N$ with $S^{\prime} \subsetneq S$, and associated feasible sets $Y_{S} \subset X_{S}$ and $Y_{S^{\prime}} \subset X_{S^{\prime}}$ where $Y_{S^{\prime}}$ is the projection of $Y_{S}$ onto $X_{S^{\prime}}$, we say that there is a vertical conflict of interest if there exists $y \in$ $B_{S}\left(x, Y_{S}\right)$ such that $y \notin B_{S^{\prime}}\left(\left(y_{S}, x_{-S}\right), Y_{S^{\prime}}\right)$.

In the SNE definition above, we have $Y_{S}=X_{S}$ for all $S \in N$. Accordingly, using the notation for SNE, a vertical conflict of interest occurs if there is $y \in B_{S}(x)$ such that $y \notin B_{S^{\prime}}\left(y_{S}, x_{-S}\right)$. The choice of $Y_{S}$ may vary for other equilibrium concepts.

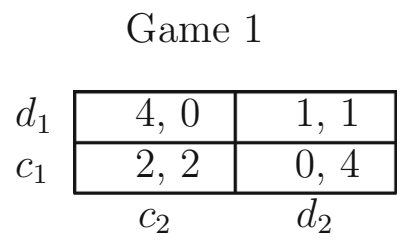

By definition, a vertical conflict of interest arises between a coalition and one of its proper subcoalitions. To illustrate, consider a Prisoner's Dilemma (see Game 1). Take the grand coalition $N$, and observe that $B_{N}(X)$ includes all strategy profiles except $\left(d_{1}, d_{2}\right)$, since it is the only strategy profile that is strictly Pareto dominated by another. Consider any $y \in B_{N}(X)$, say $\left(c_{1}, d_{2}\right)$, and further observe that when Player 2 chooses his or her strategy accordingly, i.e., when he or she chooses $d_{2}$, it is not a rational response for Player 1 to choose $c_{1}$, so that $\left(c_{1}, \cdot\right) \notin B_{1}\left(\cdot, d_{2}\right)$. This is, by definition, a vertical conflict of interest. To put it differently, in this particular case, Player 1 faces a conflict of interest between coalitional rationality required by the grand coalition and his or her own individual rationality. Further note that for games with more players, the same type of conflict may arise between a coalition of at least three players and its subcoalitions of multiple players.

Vertical conflicts of interest can be eliminated. In a two-player game, for example, no vertical conflict of interest arises if the grand coalition respects the rationality of each player by restricting itself to the set of individually rational strategy profiles (i.e., the set of Nash equilibria). More generally, no vertical conflict of interest would arise if each coalition respects the rationality of its proper subcoalitions by restricting itself to the strategy profiles from which none of its subcoalitions have an incentive to deviate.

As discussed below, this idea is closely related to "self-enforceability" in CPNE. We can formalize the idea as follows. For a set of strategy profiles $Y \subset X$ and a coalition $S \subset N$, define 


$$
[Y]_{S}=\left\{y_{S} \in X_{S} \mid \exists y_{-S} \in X_{-S}:\left(y_{S}, y_{-S}\right) \in Y\right\} .
$$

as a projection of $Y$ onto $X_{S}$. Take any $x \in X$. For any $i \in N$, define $R_{i}(x) \equiv B_{i}(x)=B_{i}\left(x, X_{i}\right)$. For any coalition $S \subset N$ with $|S|=2$, define

$$
E_{S}(x)=\left\{y \in X \mid\left(y_{S}, x_{-S}\right) \in \bigcap_{i \in S} R_{i}\left(\left(y_{S}, x_{-S}\right)\right)\right\} .
$$

The set $\left[E_{S}(x)\right]_{S}$ can be interpreted as the set of Nash equilibria of the reduced game played by coalition $S$, given that the actions of the other players are fixed to $x_{-S}$. Then, define

$$
R_{S}(x)=B_{S}\left(x,\left[E_{S}(x)\right]_{S}\right)
$$

The projection of this set onto $X_{S}$ is the set of rational responses of coalition $S$ among the strategy profiles that its members can jointly reach and that are rational for all of its members. In other words, coalition $S$ restricts itself to the strategy profiles "acceptable" to all of its members.

Likewise, for any coalition $S \subset N$ with $|S|=3$, define

$$
\begin{gathered}
E_{S}(x)=\left\{y \in X \mid\left(y_{S}, x_{-S}\right) \in \bigcap_{C \subsetneq S} R_{C}\left(\left(y_{S}, x_{-S}\right)\right)\right\} . \\
R_{S}(x)=B_{S}\left(x,\left[E_{S}(x)\right]_{S}\right) .
\end{gathered}
$$

Using (2.1) and (2.2) inductively, define $E_{S}(\cdot)$ and $R_{S}(\cdot)$ for $S \subset N$ with $|S|=4,5, \ldots,|N|$. Finally, for any strategy profile $x \in X$, define

$$
R(x)=\bigcap_{S \subset N} R_{S}(x) .
$$

A fixed point of the correspondence $R$ turns out to be a CPNE:

Proposition 2.2 A strategy profile $x \in X$ is a coalition-proof Nash equilibrium if and only if $x \in R(x)$.

Proof See the Appendix.

Although the definition of CPNE eliminates vertical conflicts of interest, there may still be what we call a "horizontal conflict of interest".

Definition 2.6 Given a strategy profile $x \in X$, two coalitions $S, S^{\prime} \subset N$ with $S \cap S^{\prime} \neq \varnothing$, and non-empty associated feasible sets $Y_{S} \subset X_{S}$ and $Y_{S^{\prime}} \subset X_{S^{\prime}}$ with the same projections onto $X_{S \cap S^{\prime}}$, we say that there is a horizontal conflict of interest if $B_{S}\left(x, Y_{S}\right) \cap B_{S^{\prime}}\left(x, Y_{S^{\prime}}\right)=\varnothing$.

Using the notation for CPNE, a horizontal conflict of interest occurs when $R_{S}(x) \cap R_{S^{\prime}}(x)=\varnothing$. In this case, $Y_{S}=\left[E_{S}(x)\right]_{S}$ for each $S \subset N$. 


\section{Game 2}

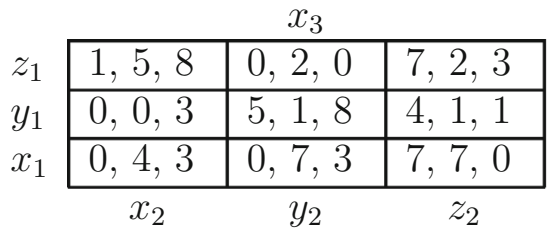

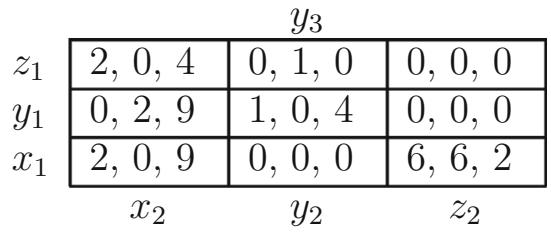

A horizontal conflict of interest is likely to arise between two coalitions with a non-empty intersection such that one coalition does not contain the other. And, this becomes possible as soon as the game has three players. Consider Game 2 as an illustrative example. For coalitions $S=\{1,2\}$ and $S^{\prime}=\{2,3\}$, we have

$$
\begin{aligned}
Y_{S} & =\left[E_{S}\left(\left(y_{1}, y_{2}, x_{3}\right)\right)\right]_{S}=\left\{\left(z_{1}, x_{2}, \cdot\right),\left(y_{1}, y_{2}, \cdot\right),\left(x_{1}, z_{2}, \cdot\right)\right\} \\
Y_{S^{\prime}} & =\left[E_{S^{\prime}}\left(\left(y_{1}, y_{2}, x_{3}\right)\right)\right]_{S^{\prime}}=\left\{\left(\cdot, x_{2}, y_{3}\right),\left(\cdot, y_{2}, x_{3}\right),\left(\cdot, z_{2}, x_{3}\right)\right\}
\end{aligned}
$$

Since the projection of either set onto $X_{S \cap S^{\prime}}$ turns out to be $X_{2}$, we verify the conditions in Definition 2.6. Now, these two coalitions have the following disjoint rational responses: $R_{S}\left(\left(y_{1}, y_{2}, x_{3}\right)\right)=\left\{\left(x_{1}, z_{2}, \cdot\right)\right\}$ and $R_{S^{\prime}}\left(\left(y_{1}, y_{2}, x_{3}\right)\right)=\left\{\left(\cdot, x_{2}, y_{3}\right)\right\}$. The interpretation is that Player 2 belongs to both coalitions $S$ and $S^{\prime}$, and although the player has the same strategies available, each coalition requires him or her to behave differently than the other coalition does. In other words, Player 2 faces a horizontal conflict of interest because he or she has no way of choosing an action that simultaneously meets the requirements of both coalitions. ${ }^{8}$

\subsection{A new equilibrium refinement}

In this section, we define a novel equilibrium notion such that there is no vertical or horizontal conflict of interest within its analysis. It is already discussed above that CPNE eliminates any vertical conflict of interest. To avoid horizontal conflicts of interest that arise between coalitions with non-empty intersections, one could easily restrict coalition formation in such a way that all active non-singleton coalitions are mutually disjoint. We have no need, however, to impose such a restrictive requirement. If any active coalition is a proper subset of another active coalition, those coalitions would still be unencumbered by horizontal conflicts of interest. To consider such collections of coalitions, we introduce the following definition.

Definition 2.7 An organization $\mathcal{O}=\left\{P_{0}, P_{1}, \ldots, P_{k}\right\}$ is an ordered collection of partitions of $N$ such that $P_{0}=\{\{1\}, \ldots,\{|N|\}\}$ and for any $i \in\{1, \ldots, k\}, P_{i-1}$ is finer than $P_{i}$.

\footnotetext{
${ }^{8}$ It can be argued that the idea of horizontal conflict of interest is not captured when $B_{S}\left(x, Y_{S}\right)$ is an empty set. However, given that such a case is realized only when $Y_{S}$ is an infinite set where each element is Pareto dominated by another element, we choose not to make further considerations in the definition for the sake of brevity.
} 
For a given organization $\mathcal{O}=\left\{P_{0}, P_{1}, \ldots, P_{k}\right\}$, define

$$
\mathscr{S}^{\mathcal{O}}=\{S \subset N \mid \exists P \in \mathcal{O} \text { such that } S \in P\}
$$

as the collection of active coalitions in that organization.

Remark 2.1 Let $\mathscr{S} \subset 2^{N}$ and $\{\{1\}, \ldots,\{|N|\}\} \subset \mathscr{S}$. Now, there exists an organization $\mathcal{O}$ such that $\mathscr{S}=\mathscr{S}^{\mathcal{O}}$ if and only if for any $A, B \in \mathscr{S}$, we have one of the following three properties: (i) $A \cap B=\varnothing$; (ii) $A \subset B$; or (iii) $B \subset A$.

Given a partition $P \in \mathcal{O}$, let $P_{-}$be the coarsest partition in $\mathcal{O}$ that is finer than $P$ and let $P_{+}$be the finest partition in $\mathcal{O}$ that is coarser than $P$. To put it differently, $P_{-}$is the layer just below $P$ and $P_{+}$is the layer just above $P$. Furthermore, given a partition $P \in \mathcal{O}$ and a coalition $S \in P$, define a suborganization $\mathcal{O}_{S \in P}$ as an ordered collection $\left\{P_{0}^{\prime}, P_{1}^{\prime}, \ldots, P_{-}^{\prime}\right\}$ of partitions of $S$ such that for each partition $P_{t}^{\prime}$ therein, we have $P_{t}^{\prime} \subset P_{t}$. Finally, let

$$
\rho^{\mathcal{O}}(S, P)=\left\{C \in \mathscr{S}^{\mathcal{O}} \mid C \in P_{-} \text {and } C \subset S\right\}
$$

be the coarsest partition in $\mathcal{O}_{S \in P}$, which is indeed a partition of $S$.

For a concrete example, consider the organization illustrated in Fig. 1. Let $\mathcal{O}=$ $\left\{P_{0}, P_{1}, P_{2}, P_{3}\right\}$ where

$$
\begin{aligned}
& P_{0}=\{\{1\},\{2\},\{3\},\{4\},\{5\},\{6\},\{7\},\{8\},\{9\},\{10\},\{11\}\}, \\
& P_{1}=\{\{1,2,3\},\{4,5\},\{6,7,8\},\{9,10\},\{11\}\}, \\
& P_{2}=\{\{1,2,3,4,5\},\{6,7,8\},\{9,10\},\{11\}\}, \text { and } \\
& P_{3}=\{\{1,2,3,4,5,6,7,8\},\{9,10,11\}\} .
\end{aligned}
$$

Moreover, if we consider $S=\{1,2,3,4,5,6,7,8\}$ and $P=P_{3}$, then the corresponding suborganization is $\mathcal{O}_{S \in P}=\left\{P_{0}^{\prime}, P_{1}^{\prime}, P_{-}^{\prime}\right\}$ such that

$$
\begin{aligned}
& P_{0}^{\prime}=\{\{1\},\{2\},\{3\},\{4\},\{5\},\{6\},\{7\},\{8\}\}, \\
& P_{1}^{\prime}=\{\{1,2,3\},\{4,5\},\{6,7,8\}\}, \text { and } \\
& P_{-}^{\prime}=\{\{1,2,3,4,5\},\{6,7,8\}\} .
\end{aligned}
$$

We are now ready to present our new equilibrium refinement. Consider a normal form game $\Gamma$ and an organization $\mathcal{O}=\left\{P_{0}, P_{1}, \ldots, P_{k}\right\}$. Take any strategy profile $x \in X$. For any player $i \in N$, define $R_{i}^{\mathcal{O}}(x) \equiv B_{i}(x)=B_{i}\left(x, X_{i}\right)$. Then for any coalition $S \in P_{1}$, the sets $E_{S}^{\mathcal{O}}(x)$ and $R_{S}^{\mathcal{O}}(x)$ are defined as follows:

$$
\begin{aligned}
& E_{S}^{\mathcal{O}}(x)=\left\{y \in X \mid\left(y_{S}, x_{-S}\right) \in \bigcap_{\{i\} \in \rho^{\mathcal{O}}(S, P)} R_{i}^{\mathcal{O}}\left(\left(y_{S}, x_{-S}\right)\right)\right\} \\
& R_{S}^{\mathcal{O}}(x)=B_{S}\left(x,\left[E_{S}^{\mathcal{O}}(x)\right]_{S}\right)
\end{aligned}
$$

Similar to the formulation of CPNE above, $R_{S}^{\mathcal{O}}(x)$ is the set of rational responses of coalition $S$ among the strategy profiles that its members can jointly reach and that are rational for all of its members. In other words, coalition $S$ restricts itself to the 


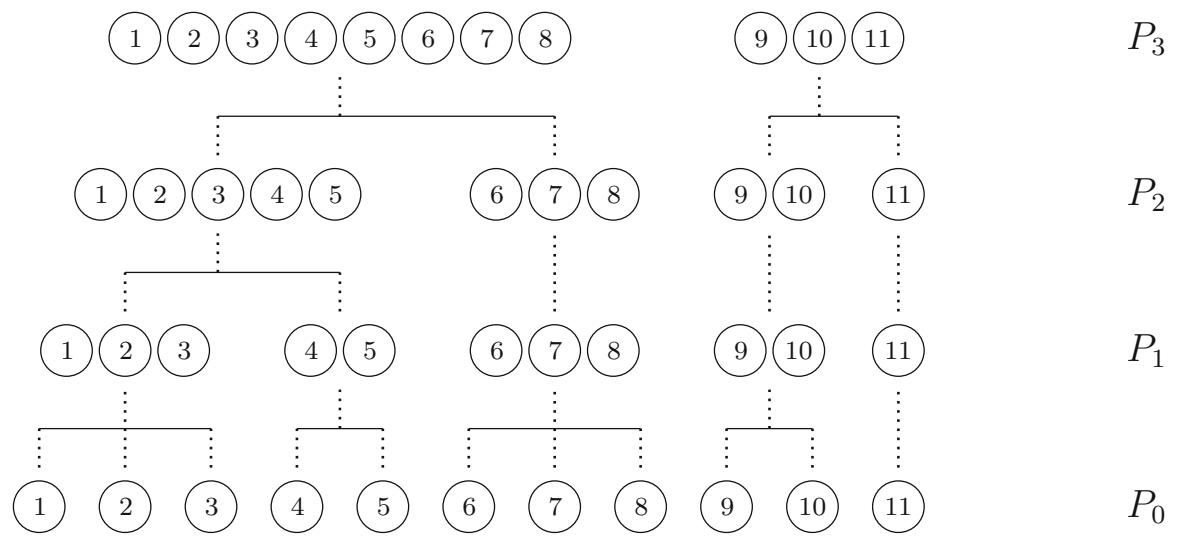

Fig. 1 Example of an organization

strategy profiles "acceptable" to all of its members. Moreover, for any coalition $S \in \mathscr{S}^{\mathcal{O}} \backslash P_{1}$, the sets $E_{S}^{\mathcal{O}}(x)$ and $R_{S}^{\mathcal{O}}(x)$ are inductively defined as follows:

$$
\begin{aligned}
& E_{S}^{\mathcal{O}}(x)=\left\{y \in X \mid\left(y_{S}, x_{-S}\right) \in \bigcap_{C \in \rho^{\mathcal{O}}(S, P)} R_{C}^{\mathcal{O}}\left(\left(y_{S}, x_{-S}\right)\right)\right\} \\
& R_{S}^{\mathcal{O}}(x)=B_{S}\left(x,\left[E_{S}^{\mathcal{O}}(x)\right]_{S}\right)
\end{aligned}
$$

The latter is the set of rational responses of coalition $S$ among the strategy profiles that its members can jointly reach and that are rational for all subcoalitions of $\mathrm{S}$. In other words, coalition $S$ restricts itself to the strategy profiles acceptable to all of its subcoalitions. And finally, for any $x \in X$, define

$$
R^{\mathcal{O}}(x)=\bigcap_{S \in \mathscr{S}^{\mathcal{O}}} R_{S}^{\mathcal{O}}(x) .
$$

We say that a strategy profile $x \in X$ is an $\mathcal{O}$-organizational Nash equilibrium, or simply an organizational Nash equilibrium, if it is a fixed point of the correspondence $R^{\mathcal{O}}$, i.e., if $x \in R^{\mathcal{O}}(x)$.

We first note that $\mathrm{ONE}$ is indeed a refinement of Nash equilibrium.

Proposition 2.3 For any normal form game $\Gamma$ and any organization $\mathcal{O}$,

$$
\mathrm{ONE}^{\mathcal{O}}(\Gamma) \subset \mathrm{NE}(\Gamma)
$$

\section{Proof Omitted.}

Given two organizations, the respective equilibrium sets may turn out to be very different. Yet, defining two organizations $\mathcal{O}$ and $\mathcal{O}^{\prime}$ to be equivalent if $\mathscr{S}^{\mathcal{O}}=\mathscr{S}^{\mathcal{O}^{\prime}}$, we show that the sets of ONE coincide for equivalent organizations. 
Remark 2.2 Given a normal form game $\Gamma$ and two equivalent organizations $\mathcal{O}, \mathcal{O}^{\prime}$ :

$$
\mathrm{ONE}^{\mathcal{O}}(\Gamma)=\mathrm{ONE}^{\mathcal{O}^{\prime}}(\Gamma) \text {. }
$$

Proof Consider any coalition $S \in \mathscr{S}^{\mathcal{O}}$. Since $\mathcal{O}$ and $\mathcal{O}^{\prime}$ are equivalent, $S \in \mathscr{S}^{\mathcal{O}^{\prime}}$. Moreover for each subcoalition $C \subset S$, if $C \in \mathscr{S}^{\mathcal{O}}$, then $C \in \mathscr{S}^{\mathcal{O}^{\prime}}$. This implies that in organizations $\mathcal{O}$ and $\mathcal{O}^{\prime}$, the coalition $S$ considers the rational responses of the same subcoalitions when making a joint decision. Then $R_{S}^{\mathcal{O}} \equiv R_{S}^{\mathcal{O}^{\prime}}$. Since $\mathrm{S}$ is arbitrarily chosen, it follows that $R^{\mathcal{O}} \equiv R^{\mathcal{O}^{\prime}}$. Hence the sets of ONE coincide.

We now focus on vertical and horizontal conflicts of interest. Although the former result in Proposition 2.4 is also valid for CPNE, the latter is only valid for ONE. The reason is that CPNE eliminates only vertical conflicts of interest, whereas ONE is also free of horizontal conflicts of interest.

Proposition 2.4 For any normal form game $\Gamma$ and any organization $\mathcal{O}$, there exists neither (i) vertical nor (ii) horizontal conflicts of interest within the analysis of ONE. Formally, (i) given two coalitions $S, S^{\prime} \in \mathscr{S}^{\mathcal{O}}$ with $S^{\prime} \subsetneq S$, and for any $x \in X$, if $\left(y_{S}, \cdot\right) \in R_{S}^{\mathcal{O}}(x)$, then $\left(y_{S^{\prime}}, \cdot\right) \in R_{S^{\prime}}^{\mathcal{O}}\left(y_{S}, x_{-S}\right)$; and (ii) given two coalitions $S, S^{\prime} \in \mathscr{S}^{\mathcal{O}}$ with $S \cap S^{\prime} \neq \varnothing$, and for any $x \in X$, we have $R_{S}^{\mathcal{O}}(x) \cap R_{S^{\prime}}^{\mathcal{O}}(x) \neq \varnothing$.

Proof For $(i)$, consider any coalition $S \in P_{1}$ and set $S^{\prime}=\{i\}$ for some $i \in S$. Take any $x \in X$ and any $\left(y_{S}, \cdot\right) \in R_{S}^{\mathcal{O}}(x)$. Noting that $R_{S}^{\mathcal{O}}(x)=B_{S}\left(x,\left[E_{S}^{\mathcal{O}}(x)\right]_{S}\right)$ by definition, we find that

$$
\left(y_{S}, \cdot\right) \in \bigcap_{i \in S} R_{i}^{\mathcal{O}}\left(\left(y_{S}, x_{-S}\right)\right) .
$$

This implies that $\left(y_{i}, \cdot\right) \in R_{i}^{\mathcal{O}}\left(y_{S}, x_{-S}\right)$. Now, consider any coalition $S \in P_{2}$. If $S^{\prime} \subset S$ is a singleton, then the result similarly follows. If not, then $S^{\prime} \in P_{1}$. Take any $x \in X$ and any $\left(y_{S}, \cdot\right) \in R_{S}^{\mathcal{O}}(x)$. Using the definition of $R_{S}^{\mathcal{O}}(x)$ once again, we find that

$$
\left(y_{S}, \cdot\right) \in \bigcap_{\substack{C \subsetneq S \\ C \in P_{0} \cup P_{1}}} R_{C}^{\mathcal{O}}\left(\left(y_{S}, x_{-S}\right)\right) .
$$

This implies that $\left(y_{S^{\prime}}, \cdot\right) \in R_{S^{\prime}}^{\mathcal{O}}\left(y_{S}, x_{-S}\right)$. The rest follows recursively.

As for (ii), consider two coalitions $S, S^{\prime} \in \mathscr{S}^{\mathcal{O}}$ such that $S \cap S^{\prime} \neq \varnothing$. By the definition of organizations, we know that one is a subset of the other, and the proof concludes with the observation that each $R_{S}^{\mathcal{O}}$ concerns only the relevant part of the strategy profiles for $S$; i.e., if $\left(y_{S}, y_{-S}\right) \in R_{S}^{\mathcal{O}}(x)$, then for every $x_{-S}^{\prime} \in X_{-S}:\left(y_{S}, x_{-S}^{\prime}\right) \in R_{S}^{\mathcal{O}}(x)$.

According to the definition of SNE, any coalition of players can jointly deviate to any of their joint strategy profiles. On the other hand, our organizational refinement restricts the set of coalitions that can deviate and the set of strategy profiles to which 
a particular coalition can deviate. Accordingly, our notion of ONE turns out to be weaker than the notion of SNE. ${ }^{9}$

Proposition 2.5 For any normal form game $\Gamma$ and any organization $\mathcal{O}$,

$$
\operatorname{SNE}(\Gamma) \subset \operatorname{ONE}^{\mathcal{O}}(\Gamma) .
$$

Proof Take any $x^{*} \in \operatorname{SNE}(\Gamma)$. Suppose that $x^{*}$ is not an $\mathcal{O}$-organizational Nash equilibrium of $\Gamma$. We then see that there should exist some partition(s) $P_{t} \in \mathcal{O}$ such that there exists some $S \in P_{t}$ satisfying $x_{S}^{*} \notin R_{S}^{\mathcal{O}}\left(x^{*}\right)$. We take the one with the smallest $t$ and denote it by $\bar{P}$. The corresponding coalition is denoted by $\bar{S}$.

Then for every $S^{\prime} \in \bar{P}_{-}$with $S^{\prime} \subset \bar{S}$ :

$$
x_{S^{\prime}}^{*} \in \mathrm{ONE}^{\mathcal{O}_{S^{\prime} \in \bar{P}_{-}}}\left(\left.\Gamma_{S^{\prime}}\right|_{x_{-S^{\prime}}^{*}}\right) .
$$

We thus obtain $y_{\bar{S}} \in X_{\bar{S}}$ such that

$$
\begin{aligned}
& \text { (i) } \forall i \in \bar{S}: u_{i}\left(y_{\bar{S}}, x_{-\bar{S}}^{*}\right)>u_{i}\left(x^{*}\right) \text { and } \\
& \text { (ii) } \forall S^{\prime} \in \bar{P}_{-} \text {with } S^{\prime} \subset \bar{S}: y_{S^{\prime}} \in \mathrm{ONE}^{\mathcal{O}_{S^{\prime} \in \bar{P}_{-}}}\left(\left.\Gamma_{S^{\prime}}\right|_{x_{-S^{\prime}}^{*}}\right) .
\end{aligned}
$$

We conclude that $x_{\bar{S}}^{*}$ is not Pareto optimal for $\bar{S}$, given that the complementary coalition chooses $x_{-S^{*}}^{*}$. This is a contradiction; which completes the proof that $x^{*}$ is an $\mathcal{O}$-organizational Nash equilibrium.

\subsection{Illustrative examples}

From Proposition 2.5, we understand the relation between the predictions of ONE and SNE. In this section, we consider two examples of normal form games to better understand how ONE refines the set of Nash equilibria and how the predictions of ONE differ from the predictions of CPNE. We will further discuss the insights gained from these equilibrium analyses later, in our Concluding Remarks.

We first revisit the three-player normal form game from Sect. 2.2, which was utilized to illustrate horizontal conflicts of interest (see Game 2). This normal form game has three Nash equilibria: $\left(z_{1}, x_{2}, x_{3}\right),\left(y_{1}, y_{2}, x_{3}\right)$, and $\left(x_{1}, z_{2}, y_{3}\right)$. The coalition $\{1,2\}$ deviates from the first and the second, the coalition $\{2,3\}$ deviates from the second and the third, and the coalition $\{1,3\}$ deviates from the first and the third. Further note that all these deviations are self-enforcing. Therefore, the game possesses no SNE or CPNE.

In Game 2, when we consider

\footnotetext{
9 This also implies that an ONE exists for every normal form game that possesses a SNE.
} 


$$
\begin{aligned}
& \mathcal{O}_{1}=\left\{P_{0},\{\{1,2\},\{3\}\},\{N\}\right\}, \\
& \mathcal{O}_{2}=\left\{P_{0},\{\{1\},\{2,3\}\},\{N\}\right\}, \\
& \mathcal{O}_{3}=\left\{P_{0},\{\{1,3\},\{2\}\},\{N\}\right\} ;
\end{aligned}
$$

we find the following sets of ONE:

$$
\begin{aligned}
& \operatorname{ONE}^{\mathcal{O}_{1}}(\cdot)=\left\{\left(x_{1}, z_{2}, y_{3}\right)\right\}, \\
& \mathrm{ONE}^{\mathcal{O}_{2}}(\cdot)=\left\{\left(z_{1}, x_{2}, x_{3}\right)\right\}, \\
& \mathrm{ONE}^{\mathcal{O}_{3}}(\cdot)=\left\{\left(y_{1}, y_{2}, x_{3}\right)\right\} .
\end{aligned}
$$

The arguments are as follows: In $O_{1}$, the only active two-player coalition is $\{1,2\}$. This coalition deviates from $\left(z_{1}, x_{2}, x_{3}\right)$ and $\left(y_{1}, y_{2}, x_{3}\right)$, but not from $\left(x_{1}, z_{2}, y_{3}\right)$. Neither of the other two-player coalitions can deviate from these strategy profiles, since they are not active. Noting that every Nash equilibrium is Pareto optimal for the grand coalition, we conclude that $\left(x_{1}, z_{2}, y_{3}\right)$ is the unique coalitionally stable outcome for this particular organization. For $\mathrm{O}_{2}$ and $\mathrm{O}_{3}$, similar arguments apply.

Note here that, as the example above illustrates, it may be the case that each Nash equilibrium is supported by some organizational structure as the unique coalitionally stable outcome. However, this does not necessarily hold for all normal form games, given that the notions of ONE and CPNE coincide in two-player games.

Remark 2.3 ONE and CPNE coincide in a two-player normal form game, because the only non-trivial organization includes all possible coalitions.

We now provide an example in which the non-empty sets of CPNE and ONE are disjoint (see Game 3). This observation implies that one equilibrium set does not necessarily include the other.

\section{Game 3}
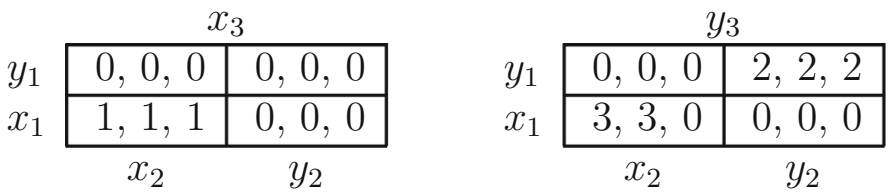

Note that there are two Nash equilibria: $\left(x_{1}, x_{2}, x_{3}\right)$ and $\left(y_{1}, y_{2}, y_{3}\right)$. And there exists a unique CPNE: $\left(x_{1}, x_{2}, x_{3}\right)$. This is true, because the coalition $\{1,2\}$ makes a self-enforcing deviation from $\left(y_{1}, y_{2}, y_{3}\right)$; whereas the subcoalition $\{1,2\}$ blocks the deviation of the grand coalition from $\left(x_{1}, x_{2}, x_{3}\right)$ to $\left(y_{1}, y_{2}, y_{3}\right)$, as the subcoalition would further deviate from $\left(y_{1}, y_{2}, y_{3}\right)$. On the other hand, if we analyze ONE of this game for the organization $\left\{P_{0},\{\{1\},\{2,3\}\},\{N\}\right\}$, the unique ONE turns out to be $\left(y_{1}, y_{2}, y_{3}\right)$. This result follows because now that the coalition $\{1,2\}$ is inactive, they would not deviate from $\left(y_{1}, y_{2}, y_{3}\right)$ and could not block the deviation of the grand 
coalition from $\left(x_{1}, x_{2}, x_{3}\right)$ to $\left(y_{1}, y_{2}, y_{3}\right)$. By a similar reasoning, the unique ONE of this game would be $\left(y_{1}, y_{2}, y_{3}\right)$ also for the organizations $\left\{P_{0},\{\{1,3\},\{2\}\},\{N\}\right\}$ and $\left\{P_{0},\{N\}\right\}$. Note also how this example highlights the importance and usefulness of our organizational refinement. Apparently, the unique ONE strictly Pareto dominates the unique CPNE.

\section{The results}

\subsection{The existence of equilibrium}

Although the formulation of organizational refinements does not suffer from the problems embedded in vertical and horizontal conflicts of interest, a normal form game might not have an ONE for some organizations. For example, Game 4 has a unique Nash equilibrium: $x=\left(x_{1}, x_{2}, x_{3}\right)$. However, considering the organization $\mathcal{O}=\left\{P_{0},\{\{1,2\},\{3\}\}\right\}, \quad$ we have $R_{1}^{\mathcal{O}}(x)=\left(x_{1}, \cdot, \cdot\right), \quad R_{2}^{\mathcal{O}}(x)=\left(\cdot, x_{2}, \cdot\right), \quad$ and $R_{\{1,2\}}^{\mathcal{O}}(x)=\left(y_{1}, y_{2}, \cdot\right)$. Then $R^{\mathcal{O}}(x)=\varnothing$. The non-existence of ONE follows.

\section{Game 4}

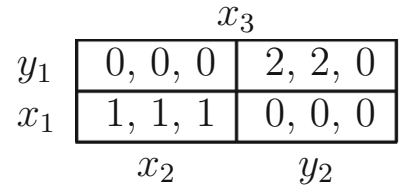

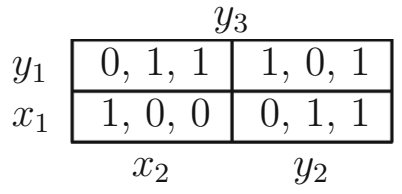

The foregoing observation naturally compels us to find classes of normal form games where an ONE exists. In this section of the paper, we prove the existence of our organizational refinement in a subclass of games with strategic complementarities (see Topkis 1998; Amir 2005; Vives 2005, among others). ${ }^{10}$ The following are the definitions that will be utilized throughout this section.

A set is a lattice if it contains the supremum and the infimum of every pair of its elements. A lattice is complete if each non-empty subset has a supremum and an infimum. ${ }^{11}$ Moreover, a subset $Y$ of a lattice $X$ is a subcomplete sublattice of $X$ if for every non-empty subset $Y^{\prime}$ of $Y$, the supremum of $Y^{\prime}$ and the infimum of $Y^{\prime}$ exist and are contained in $Y$. Let $X$ be a lattice and $T$ be a partial order. For any $x, y \in X, x \vee y$ denotes their lowest upper bound and $x \wedge y$ denotes their greatest lower bound. A function $f: X \rightarrow \mathbb{R}$ is called quasi-supermodular if for every

\footnotetext{
${ }^{10}$ Games with strategic complemetarities are commonly used in the literature both for the existence of Nash equilibrium (see Zhou 1994; Echenique 2005; Calciano 2007; Keskin et al. 2014, among others) and for the existence of some of the refinements of Nash equilibrium; such as minimally altruistic Nash equilibrium (see Karagözoğlu et al. 2013), perfect equilibrium (see Carbonell-Nicolau and McLean 2015), and strong Berge equilibrium (see Keskin and Sağlam 2014).

11 Note that a complete lattice $X$ is compact in its interval topology which is the topology generated by taking the closed intervals, $[y, z]=\{x \in X: y \leq x \leq z\}$ with $y, z \in X$ as a subbasis of closed sets (see Birkhoff 1967).
} 
$x, y \in X: f(x) \geq f(x \wedge y)$ implies that $f(x \vee y) \geq f(y)$ and $f(x)>f(x \wedge y)$ implies that $f(x \vee y) \geq f(y)$. We say that a function $f: X \times T \rightarrow \mathbb{R}$ satisfies the single crossing property in $(x, t)$ if for every $x, x^{\prime} \in X$ and $t, t \in T$ with $x>x^{\prime}$ and $t>t^{\prime}$ : $f\left(x, t^{\prime}\right) \geq f\left(x^{\prime}, t^{\prime}\right)$ implies that $f(x, t) \geq f\left(x^{\prime}, t\right)$ and $f\left(x, t^{\prime}\right)>f\left(x^{\prime}, t^{\prime}\right)$ implies that $f(x, t)>f\left(x^{\prime}, t\right)$.

The following definition of games with strategic complementarities is provided by Milgrom and Shannon (1994) and Milgrom and Roberts (1996).

Definition 3.1 A normal form game $\Gamma$ is a game with strategic complementarities if for every $i \in N$ : (i) $X_{i}$ is a non-empty complete lattice; (ii) $u_{i}$ is upper semicontinuous in $x_{i}$ and continuous in $x_{-i}$; and (iii) $u_{i}$ is quasi-supermodular in $x_{i}$ and has the single crossing property in $\left(x_{i}, x_{-i}\right)$.

Milgrom and Shannon (1994) show that the smallest and the largest serially undominated strategy profiles ${ }^{12}$ exist in a game with strategic complementarities and are, respectively, the smallest and the largest Nash equilibria of the game (see their Theorem 12). Furthermore, as shown by Milgrom and Roberts (1996), an additional monotonicity assumption would suffice for the existence of CPNE in a subclass of games with strategic complementarities. In particular, the authors assume that each utility function $u_{i}$ is non-decreasing/non-increasing in $x_{-i}$ (see their Theorem 2). In the following, we prove the existence of our organizational refinement by weakening this monotonicity assumption.

Proposition 3.1 Consider a game with strategic complementarities $\Gamma$ and any organization $\mathcal{O}$. Assume that for every $i \in N$ and every $S \in \mathscr{S}^{\mathcal{O}}$ that includes $i$ : either $(i) u_{i}$ is non-decreasing in $x_{S \backslash\{i\}}$, or (ii) $u_{i}$ is non-increasing in $x_{S \backslash\{i\}}$. Then, there exists an $\mathcal{O}$-organizational Nash equilibrium for this game.

Proof Assume case ( $i$ ) above and consider the largest Nash equilibrium of the game, denoted by $x^{*}$. As we know from Milgrom and Shannon (1994), $x^{*}$ is also the largest serially undominated strategy profile in this game. Consider any coalition $S \in P_{1}$ and the corresponding reduced game $\left.\Gamma_{S}\right|_{x_{-S}^{*}}=\left(S,\left(X_{i}\right)_{i \in S},\left(v_{i}\right)_{i \in S}\right)$. By definition, this is a reduced game with strategic complementarities in which each $v_{i}$ is non-decreasing in $x_{-i}$. We also know that $x_{S}^{*}$ is a Nash equilibrium for $\left.\Gamma_{S}\right|_{x_{-S}^{*}}$, which means that $x_{S}^{*}$ would survive the iterated elimination of strictly dominated strategies in the reduced game. As a matter of fact, $x_{S}^{*}$ turns out to be the largest serially undominated strategy profile in this game. ${ }^{13}$ It then follows from Milgrom and Shannon (1994) that $x_{S}^{*}$ is the largest Nash equilibrium for the reduced game. Applying Theorem A2 from Milgrom and Roberts (1996), each subcoalition $C \subset S$ prefers playing $x_{C}^{*}$ to any other strategy profile in the reduced game. Their theorem surely applies to the coalition $S$ itself. Accordingly, for any Nash equilibrium $y_{S}$ of the reduced game $\left.\Gamma_{S}\right|_{x_{-S}^{*}}$, we have for every $i \in S: u_{i}\left(y_{S}, x_{-S}^{*}\right) \leq u_{i}\left(x_{S}^{*}\right)$. Therefore, $x_{S}^{*}$ is a coalitional best response for $S$. Since $S$ is arbitrarily chosen, for every coalition $S \in P_{1}:\left(x_{S}^{*}, \cdot\right) \in R_{S}^{\mathcal{O}}\left(x^{*}\right)$.

\footnotetext{
12 A strategy profile is said to be serially undominated if it survives the iterated elimination of strictly dominated strategies.

13 The reader is referred to the Appendix for the proof of this claim.
} 
Now, consider any coalition $S^{\prime} \in P_{2}$ and the corresponding reduced game $\left.\Gamma_{S^{\prime}}\right|_{x_{-S^{\prime}}^{*}}$. Noting that $\mathcal{O}_{S^{\prime} \in P_{2}}$ is the suborganization for this coalition and considering the arguments above, we know that $x_{S^{\prime}}^{*}$ is an $\mathcal{O}_{S^{\prime} \in P_{2}}$-organizational Nash equilibrium of this game. This follows because for every member $i \in S^{\prime}:\left(x_{i}^{*}, \cdot\right) \in R_{i}^{\mathcal{O}}\left(x^{*}\right)$ as well as for every active subcoalition $C^{\prime} \subset S^{\prime}$ in this suborganization: $\left(x_{C^{\prime}}^{*}, \cdot\right) \in R_{C^{\prime}}^{\mathcal{O}}\left(x^{*}\right)$. Applying Theorem A2 from Milgrom and Roberts (1996) once again, we conclude that the coalition $S^{\prime}$ prefers playing $x_{S^{\prime}}^{*}$ to any other ONE in the reduced game $\left.\Gamma_{S^{\prime}}\right|_{x_{-S^{\prime}}^{*}}$. It similarly follows that for every coalition $S^{\prime} \in P_{2}:\left(x_{S^{\prime}}^{*}, \cdot\right) \in R_{S^{\prime}}^{\mathcal{O}}\left(x^{*}\right)$.

Finally, it recursively follows that for every coalition $S^{\prime \prime} \in \mathscr{S}^{\mathcal{O}}:\left(x_{S^{\prime \prime}}^{*}, \cdot\right) \in R_{S^{\prime \prime}}^{\mathcal{O}}\left(x^{*}\right)$. Hence, $x^{*} \in R^{\mathcal{O}}\left(x^{*}\right)$, which in turn implies that $x^{*} \in \operatorname{ONE}(\Gamma)$.

Arguments for case (ii) similarly follow.

We can demonstrate how the existence result works in Game 5.

\section{Game 5}
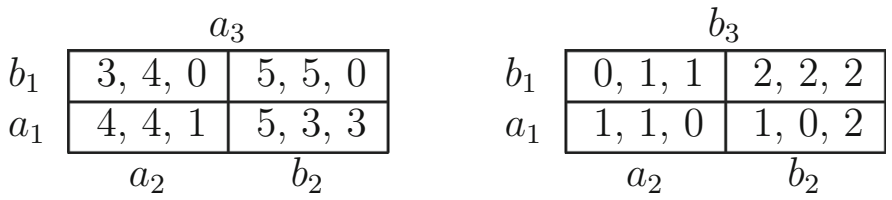

This example is a game with strategic complementarities since each utility function $u_{i}$ is quasi-supermodular in $x_{i}$ and has the single crossing property in $\left(x_{i}, x_{-i}\right)$. Furthermore, $u_{1}$ is non-decreasing in $x_{2}$ and $u_{2}$ is non-decreasing in $x_{1}$. Also note that $u_{3}$ is not monotone in $x_{1}$ or $x_{2}$, since $\left(a_{1}, b_{2}, a_{3}\right)$ yields the highest utility for Player 3. Accordingly, this normal form game satisfies the conditions of our existence result for the organization $\mathcal{O}^{*}=\left\{P_{0},\{\{1,2\},\{3\}\}\right\}$, but not for the other possible organizations. Neither does it satisfy the conditions for the existence of CPNE provided by Milgrom and Roberts (1996). There are two Nash equilibria: $\left(a_{1}, a_{2}, a_{3}\right)$ and $\left(b_{1}, b_{2}, b_{3}\right)$. Now, analyzing the set of $\mathcal{O}^{*}$-organizational Nash equilibria, we see that the coalition $\{1,2\}$ deviates from $\left(a_{1}, a_{2}, a_{3}\right)$ to $\left(b_{1}, b_{2}\right)$ when Player 3 sticks to $a_{3}$. Observing that they would not deviate from the other Nash equilibrium, we find that $\left(b_{1}, b_{2}, b_{3}\right)$ is the unique ONE for this game. We also know, meanwhile, that no CPNE exists, because $(i)$ the coalition $\{1,2\}$ still makes the aforementioned deviation and (ii) the coalition $\{1,3\}$ makes a self-enforcing deviation from $\left(b_{1}, b_{2}, b_{3}\right)$ to $\left(a_{1}, a_{3}\right)$ when Player 2 sticks to $b_{2}$. 


\section{Game 6}

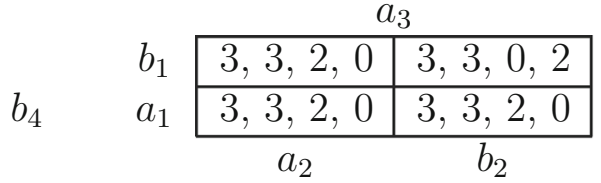

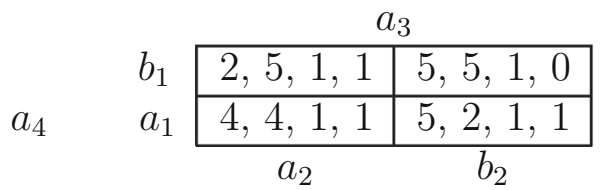

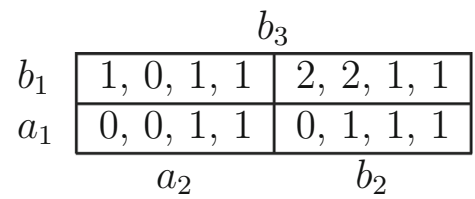

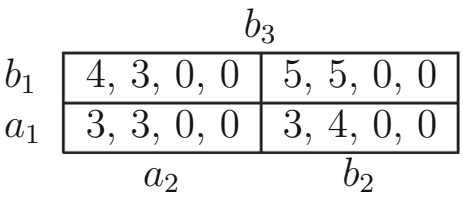

As another example satisfying the conditions of Proposition 3.1, consider the four-player normal form game, labeled as Game 6. Once again, $u_{1}$ is non-decreasing in $x_{2}$ and $u_{2}$ is non-decreasing in $x_{1}$. We can also see that neither $u_{3}$ nor $u_{4}$ is monotone in either of the other players' strategies. This game has two Nash equilibria: $\left(a_{1}, a_{2}, a_{3}, a_{4}\right)$ and $\left(b_{1}, b_{2}, b_{3}, b_{4}\right)$. Considering the organization $\mathcal{O}^{*}$ above, we note that the coalition $\{1,2\}$ deviates from $\left(a_{1}, a_{2}, a_{3}, a_{4}\right)$ to $\left(b_{1}, b_{2}\right)$ when Players 3 and 4 stick to $\left(a_{3}, a_{4}\right)$, and we find that $\left(b_{1}, b_{2}, b_{3}, b_{4}\right)$ is the unique ONE for this game. Now, if we consider another organization by adding $P_{2}=$ $\{\{1,2,3\},\{4\}\}$ into the existing organization $\mathcal{O}^{*}$, we see that an ONE ceases to exist. This is because the coalition $\{1,2,3\}$ deviates from $\left(b_{1}, b_{2}, b_{3}, b_{4}\right)$ to $\left(a_{1}, a_{2}, a_{3}\right)$ when Player 4 sticks to $b_{4}$. As we see in this particular example, adding a new coalition for which the utility functions of its members do not have a monotonicity relation as described in Proposition 3.1 might lead to the nonexistence of equilibrium.

Another interesting note regarding this example is that if one considers the organization $\left\{P_{0},\{\{1,2,3\},\{4\}\}\right\}$, then the weakly Pareto optimal Nash equilibrium $\left(a_{1}, a_{2}, a_{3}, a_{4}\right)$ is realized as the unique ONE. Accordingly, we can claim that removing small coalitions from an organization or replacing small coalitions in an organization with larger coalitions might turn out to be socially beneficial. 


\subsection{A monotonicity property}

We start with a nice result indicating that the introduced refinement structures follow in a monotonic fashion. In a normal form game, given two organizations $\mathcal{O}$ and $\mathcal{O}^{\prime}$, we say that $\mathcal{O}$ is greater than $\mathcal{O}^{\prime}$ if (i) $P \in \mathcal{O}^{\prime}$ implies that $P \in \mathcal{O}$ and (ii) $\left[P^{\prime} \in \mathcal{O}\right.$ and $\left.P^{\prime} \notin \mathcal{O}^{\prime}\right]$ implies that $P^{\prime}$ is coarser than the coarsest partition in $\mathcal{O}^{\prime}$. We now show that the equilibrium set is more refined for greater organizations. ${ }^{14}$

Proposition 3.2 For any normal form game $\Gamma$, if an organization $\mathcal{O}$ is greater than another organization $\mathcal{O}^{\prime}$, then

$$
\operatorname{ONE}^{\mathcal{O}}(\Gamma) \subset \operatorname{ONE}^{\mathcal{O}^{\prime}}(\Gamma)
$$

Proof Take any $x^{*} \in \mathrm{ONE}^{\mathcal{O}}(\Gamma)$. By definition, $x^{*} \in R^{\mathcal{O}}\left(x^{*}\right)$. This implies that for every $S \in \mathscr{S}^{\mathcal{O}}:\left(x_{S}^{*}, \cdot\right) \in R_{S}^{\mathcal{O}}\left(x^{*}\right)$. Since $\mathcal{O}$ is greater than $\mathcal{O}^{\prime}$, we know that $\mathscr{S}^{\mathcal{O}^{\prime}} \subset$ $\mathscr{S}^{\mathcal{O}}$ and that for every $S \in \mathscr{S}^{\mathcal{O}} \backslash \mathscr{S}^{\mathcal{O}^{\prime}}: \nexists S^{\prime} \in \mathscr{S}^{\mathcal{O}^{\prime}}$ such that $S \subset S^{\prime}$. Accordingly, for every $S^{\prime} \in \mathscr{S}^{\mathcal{O}^{\prime}}:\left(x_{S^{\prime}}^{*}, \cdot\right) \in R_{S^{\prime}}^{\mathcal{O}^{\prime}}\left(x^{*}\right)$. Therefore, $x^{*} \in R^{\mathcal{O}^{\prime}}\left(x^{*}\right)$; i.e., $x^{*} \in \mathrm{ONE}^{\mathcal{O}^{\prime}}(\Gamma)$. It thus follows that $\operatorname{ONE}^{\mathcal{O}}(\Gamma) \subset \mathrm{ONE}^{\mathcal{O}^{\prime}}(\Gamma)$.

This monotonicity property leads to the following observation.

Corollary 3.1 Consider a normal form game $\Gamma$ that possesses a Nash equilibrium. Take any increasing sequence of organizations $\mathcal{O}_{1}, \mathcal{O}_{2}, \ldots, \mathcal{O}_{t}$ such that $\mathcal{O}_{1}=\left\{P_{0}\right\}$ and for any $i \in\{1, \ldots, t-1\}: \mathcal{O}_{i+1}$ is greater than $\mathcal{O}_{i}$. We have

$$
\mathrm{ONE}^{\mathcal{O}_{i+1}}(\Gamma) \subset \mathrm{ONE}^{\mathcal{O}_{i}}(\Gamma)
$$

for any $i \in\{1, \ldots, t-1\}$. In addition, the sequence has a maximum organization for which the set of ONE is non-empty.

In normal form games with multiple equilibria, one can form the second layer of an organization in order to decrease the number of equilibria. If the multiplicity is still preserved, one can continue with the upper layers of the organization until it is about to become "too big" such that it will fail to take an action (or, reach an equilibrium). The corollary above indicates that the formation process stops at a unique point before an ONE ceases to exist. This is demonstrated in the following example.

Example Consider Game 7, which is a five-player normal form game with three Nash equilibria: $\left(x_{1}, x_{2}, y_{3}, y_{4}, x_{5}\right),\left(y_{1}, y_{2}, x_{3}, y_{4}, x_{5}\right),\left(y_{1}, y_{2}, y_{3}, x_{4}, x_{5}\right)$.

\footnotetext{
${ }^{14}$ It is important here that we do not compare any particular partitions when comparing two organizations. Our definition of "being greater" indicates that an organization is greater than another if the former completely preserves the structure of the latter and additionally includes coarser partitions. Alternative definitions may yield different results.
} 
Game 7

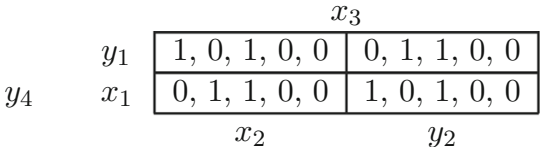

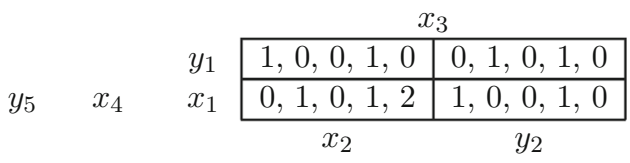

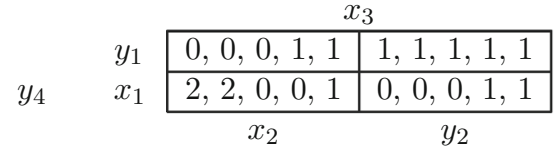

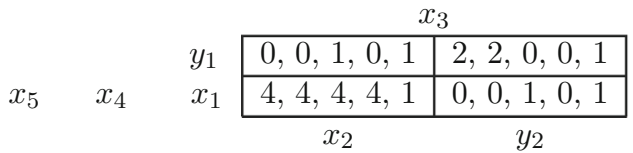

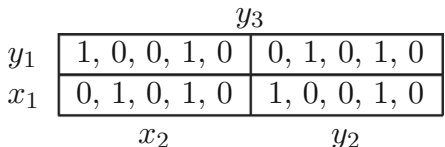

\begin{tabular}{l|c|c|}
\multicolumn{3}{c}{$y_{3}$} \\
\cline { 2 - 3 }$y_{1}$ & $1,0,1,0,0$ & $0,1,1,0,0$ \\
\cline { 2 - 3 }$x_{1}$ & $0,1,1,0,0$ & $1,0,1,0,0$ \\
\cline { 2 - 3 }$x_{2}$ & \multicolumn{2}{c}{$y_{2}$}
\end{tabular}

\begin{tabular}{c|c|c|}
\multicolumn{2}{c}{$y_{3}$} \\
\cline { 2 - 3 }$y_{1}$ & $0,0,1,0,1$ & $1,1,0,1,1$ \\
\cline { 2 - 3 }$x_{1}$ & $3,3,3,3,1$ & $0,0,1,0,1$ \\
\cline { 2 - 3 } & \multicolumn{2}{c|}{$x_{2}$}
\end{tabular}

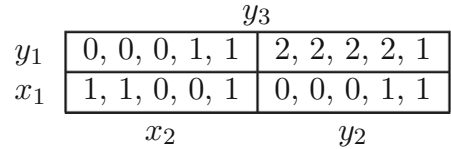

We now set

$$
\begin{aligned}
& P_{0}=\{\{1\},\{2\},\{3\},\{4\},\{5\}\}, \\
& P_{1}=\{\{1,2\},\{3\},\{4\},\{5\}\}, \\
& P_{2}=\{\{1,2,3\},\{4\},\{5\}\}, \text { and } \\
& P_{3}=\{\{1,2,3,4\},\{5\}\} .
\end{aligned}
$$

First consider $\mathcal{O}_{1}=\left\{P_{0}\right\}$. Here, the set of ONE surely coincides with the set of Nash equilibria. Next, consider $\mathcal{O}_{2}=\left\{P_{0}, P_{1}\right\}$. We see that the coalition $\{1,2\}$ deviates to $\left(x_{1}, x_{2}\right)$ when Players 3,4 , and 5 stick to $\left(x_{3}, y_{4}, x_{5}\right)$. Since there is no further deviation, $\left(y_{1}, y_{2}, x_{3}, y_{4}, x_{5}\right)$ is not coalitionally stable in the sense of ONE. And since there is no other deviation, the remaining two Nash equilibria turn out to be ONE for this game:

$$
\left(x_{1}, x_{2}, y_{3}, y_{4}, x_{5}\right),\left(y_{1}, y_{2}, y_{3}, x_{4}, x_{5}\right) \text {. }
$$

If we consider $\mathcal{O}_{3}=\left\{P_{0}, P_{1}, P_{2}\right\}$, we see that the coalition $\{1,2,3\}$ deviates to $\left(x_{1}, x_{2}, x_{3}\right)$ when Players 4 and 5 stick to $\left(x_{4}, x_{5}\right)$. Since there is no further deviation, $\left(y_{1}, y_{2}, y_{3}, x_{4}, x_{5}\right)$ is not coalitionally stable in the sense of ONE. And since there is no other deviation, there exists a unique ONE: 


$$
\left(x_{1}, x_{2}, y_{3}, y_{4}, x_{5}\right) \text {. }
$$

Finally, when we consider $\mathcal{O}_{4}=\left\{P_{0}, P_{1}, P_{2}, P_{3}\right\}$, the coalition $\{1,2,3,4\}$ deviates to $\left(x_{1}, x_{2}, x_{3}, x_{4}\right)$ when Player 5 sticks to $x_{5}$. Since there is no further deviation, the set of ONE turns out to be empty.

It is also worth noting that because of all deviations mentioned above, this fiveplayer normal form game has neither a SNE nor a CPNE. $\diamond$

The following observation utilizes a different perspective.

Corollary 3.2 Consider a normal form game $\Gamma$ that possesses a Nash equilibrium. Take any organization $\overline{\mathcal{O}}$ and any decreasing sequence of organizations $\mathcal{O}_{1}, \mathcal{O}_{2}, \ldots, \mathcal{O}_{t}$ such that $\mathcal{O}_{1}=\overline{\mathcal{O}}$, for any $i \in\{2, \ldots, t\}: \mathcal{O}_{i}$ is greater than $\mathcal{O}_{i-1}$, and $\mathcal{O}_{t}=\left\{P_{0}\right\}$. We have

$$
\mathrm{ONE}^{\mathcal{O}_{i-1}}(\Gamma) \subset \mathrm{ONE}^{\mathcal{O}_{i}}(\Gamma)
$$

for any $i \in\{2, \ldots, t\}$. In addition, the sequence has a maximum organization for which the set of ONE is non-empty.

In some normal form games, there may exist a "too big" organization that fails to take an action (or, reach an equilibrium). In such cases, it might help to dissolve all of the coalitions in the final layer of the organization and play the game as a "smaller" organization. If an ONE still does not exist, the dissolving process may continue. The corollary above indicates that the process stops at a unique point for which the normal form game possesses an ONE.

\section{Concluding remarks}

In this paper, we have studied cases in which some coalitions cannot be formed. In particular, taking an organizational structure as given, we have introduced a new refinement of Nash equilibrium and proved the existence of equilibria in a subclass of games with strategic complementarities. Moreover, through remarks and examples, we have further analyzed how our notion refines the set of Nash equilibria.

Organizational refinements can lead to many interesting and fruitful questions. First, one can investigate the robustness of equilibrium. More precisely, some Nash equilibria may remain to be an equilibrium for any given organization, whereas some others may fail to be an equilibrium as soon as any organization is formed. One can, therefore, consider the former to be the most robust and the latter to be the least robust Nash equilibrium. In that sense, any two Nash equilibria can be compared in terms of robustness to organizational deviations. Such an analysis may also provide general insights for certain classes of games.

Second, one can study the endogenous formation of organizations. There can be several methods for this exercise. Either (i) players may have pre-defined preferences over the set of coalitions or organizations that somehow induce organizational structures; or (ii) as the set of equilibria is now known for any given 
organization, players may form coalitions/organizations strategically by opting for organizational structures that yield "the best" set of equilibria. As an example for the latter method, recall Game 2 from Sect. 2.2. In that game, either of the three Nash equilibria can be captured by a certain organization. Among the three Nash equilibria, $\left(x_{1}, z_{2}, y_{3}\right)$ is Pareto optimal for the coalition $\{1,2\}$. And Players 1 and 2 are able to reach there by forming the two-player coalition, thereby blocking the formation of $\{1,3\}$ and $\{2,3\}$.

Third, one can analyze policy implications. Notice that the formation of coalitional or organizational structures does not have to be strategic (as described above). For instance, a social planner may be interested in enforcing a socially optimal organization. As an example, recall Game 3 from Sect. 2.4. Given the existence of an organization for which the unique ONE strictly Pareto dominates the unique CPNE, a social planner would prefer to forbid the formation of $\{1,2\}$.

\section{Appendix}

\section{On the Notions of SNE and CPNE}

In this part of the Appendix, we prove that the alternative definitions we provided for the notions of SNE and CPNE are equivalent to their standard definitions in the literature, also presented in Definitions 2.2 and 2.4.

We first recall our result on the notion of SNE.

Proposition 2.1. A strategy profile $x \in X$ is a strong Nash equilibrium if and only if $x \in B(x)$.

Proof Take any $x \in X$ such that $x \in B(x)$. Suppose that $x$ is not a SNE. Then $\exists S \subset N, \exists z_{S} \in X_{S}$ such that $\forall i \in S: u_{i}\left(z_{S}, x_{-S}\right)>u_{i}\left(x_{S}, x_{-S}\right)$. We then have $x \notin B_{S}(x)$; a contradiction.

Conversely, take any $x \in \operatorname{SNE}(\cdot)$. Suppose that $x \notin B(x)$. Then $\exists S \subset N$ such that $x \notin B_{S}(x)$; that is, $\exists z_{S} \in X_{S}, \forall i \in S: u_{i}\left(z_{S}, x_{-S}\right)>u_{i}\left(x_{S}, x_{-S}\right)$; a contradiction.

As for the notion of CPNE, we first prove the following lemma and then proceed to the equivalence result.

Lemma A.1 For any coalition $S \subset N$ with $|S| \geq 2$ and any strategy profile $x \in X$, we have

$$
\bigcap_{C \subsetneq S} R_{C}(x)=\bigcap_{\substack{C \subset S \\|C|=|S|-1}} R_{C}(x) .
$$

Proof It is trivial that the left-hand side is included in the right-hand side.

Conversely, take any

$$
x \in \bigcap_{\substack{C \subset S \\|C|=|S|-1}} R_{C}(x),
$$

and suppose that 


$$
x \notin \bigcap_{C \subsetneq S} R_{C}(x) .
$$

Then $\exists C \subsetneq S$ such that $x \notin R_{C}(x)$. Note that there exists $C^{\prime} \subset S$ such that $C \subset C^{\prime}$ and $\left|C^{\prime}\right|=|S|-1$. Since the definition is recursive, it follows that $x \notin R_{C^{\prime}}(x)$; a contradiction.

Here we recall our result on the notion of CPNE as well.

Proposition 2.2. A strategy profile $x \in X$ is a coalition-proof Nash equilibrium if and only if $x \in R(x)$.

Proof We prove by induction. Clearly, the statement holds when $|N|=1$. Assume that it also holds when $|N| \leq k-1$ for some $k \in \mathbb{N}$.

Consider $|N|=k$. Take any $x \in X$ such that $x \in R(x)$. And suppose that $x$ is not a CPNE. Then either $(i) x$ is not self-enforcing; or (ii) $x$ is self-enforcing but there exists another self-enforcing strategy profile $z \in R(x)$ such that $\forall i \in N: u_{i}(z)>u_{i}(x)$. If (i) is the case, then $\exists S \subsetneq N$ such that $x_{S} \notin \operatorname{CPNE}\left(\left.\Gamma_{S}\right|_{x_{-}}\right)$. But then $x \notin R_{S}(x)$ by the induction hypothesis; a contradiction. If, on the other hand, case (ii) applies, then it must be that $x \notin R_{N}(X)$; a contradiction.

Conversely, take any $x \in \mathrm{CPNE}(\cdot)$. And suppose that $x \notin R(x)$. Then either (i) $x \notin R_{N}(x)$; or (ii) $x \notin \bigcap_{S \subsetneq N} R_{S}(x)$. If case (i) applies, then there exists another strategy profile $z \in R(x)$ such that $\forall i \in N: u_{i}(z)>u_{i}(x)$. By the induction hypothesis, $z$ must be self-enforcing; a contradiction. On the other hand, if (ii) is the case, then

$$
x \notin \bigcap_{\substack{S \subset N \\|S|=|N|-1}} R_{S}(x)
$$

by Lemma A.1. By a similar reasoning, it follows for some $S \subset N$ with $|S|=$ $|N|-1$ that $x_{S}$ is not a CPNE of the corresponding reduced game. This implies that $x$ is not self-enforcing; a contradiction.

\section{About the Existence of Equilibrium}

We first note that for a player $i \in N$, a strategy $x_{i} \in X_{i}$ is strictly dominated if there exists another strategy $x_{i}^{\prime} \in X_{i}$ such that for every $x_{-i} \in X_{-i}$ :

$$
u_{i}\left(x_{i}^{\prime}, x_{-i}\right)>u_{i}\left(x_{i}, x_{-i}\right) .
$$

The following lemma is used in the proof of Proposition 3.1.

Lemma A.2 In a normal form game $\Gamma$, let $x^{*}$ be a Nash equilibrium which is also the largest serially undominated strategy profile. Then $x_{S}^{*}$ turns out to be the largest serially undominated strategy profile of the reduced game $\left.\Gamma_{S}\right|_{x_{-S}^{*}}$ for any coalition $S \subset N$.

Proof Take any coalition $S \subset N$. Note that $x_{S}^{*}$ is a Nash equilibrium of the reduced game $\left.\Gamma_{S}\right|_{x_{-S}^{*}}$. Thus, it is a serially undominated strategy profile. 
We now describe a particular procedure of iterated elimination of strictly dominated strategies: We start with $\Gamma^{0} \equiv \Gamma$. At stage 1, only Player 1's dominated strategies are eliminated. The resulting game is labeled as $\Gamma^{1}$. At stage 2, only Player 2's dominated strategies are eliminated. The resulting game is labeled as $\Gamma^{2}$. After each player has one elimination stage, we reach $\Gamma^{n}$. From stage $n+1$ onwards, the same procedure follows. More generally, for any $k \in \mathbb{N}_{0}$, only Player $i$ 's dominated strategies are eliminated in stage $i+k n$. This procedure continues until $\Gamma^{\infty}$.

Without loss of generality, assume that Player 1 is a member of $S$ and that $x_{1} \in X_{1}$ is strictly dominated by some $x_{1}^{\prime} \in X_{1}$. In the reduced game $\left.\Gamma_{S}\right|_{x_{-S}^{*}}$, we have

$$
u_{1}\left(x_{1}^{\prime}, x_{S \backslash\{1\}}, x_{-S}^{*}\right)>u_{1}\left(x_{1}, x_{S \backslash\{1\}}, x_{-S}^{*}\right)
$$

for every $x_{S \backslash\{1\}} \in X_{S \backslash\{1\}}$. This shows that $x_{1}$ remains to be strictly dominated in the reduced game.

Now, we start with $\left.\Gamma_{S}^{0} \equiv \Gamma_{S}\right|_{x_{-S}^{*}}$. At stage 1, only Player 1's dominated strategies in $\Gamma^{0}$ are eliminated. The resulting game is labeled as $\Gamma_{S}^{1}$. Notice that Player 1 may have additional dominated strategies in the reduced game, but even so, we do not eliminate those at this stage. Notice further that $\Gamma_{S}^{1}$ is a reduced game of $\Gamma^{1}$. For the next stage, if Player 2 is a member of $S$, then only Player 2's dominated strategies in $\Gamma^{1}$ are eliminated and the resulting game is labeled as $\Gamma_{S}^{2}$; but if otherwise, then we simply set $\Gamma_{S}^{2}=\Gamma_{S}^{1}$. Notice that, in either case, $\Gamma_{S}^{2}$ becomes a reduced game of $\Gamma^{2}$. This procedure eventually yields a reduced game $\Gamma_{S}^{\infty}$ of $\Gamma^{\infty}$.

Given a player $i \in S$, we know that whichever strategy $y_{i} \not \leq x_{i}^{*}$ is eliminated in some stage of the iterated elimination of strictly dominated strategies for $\Gamma$, the same strategy should be eliminated in the corresponding stage of the iterated elimination of strictly dominated strategies for $\left.\Gamma_{S}\right|_{x_{-S}^{*}}$. Also knowing that $x_{S}^{*}$ is serially undominated, we conclude that $x_{S}^{*}$ is the largest serially undominated strategy profile.

Acknowledgement We would like to thank the editor, an anonymous reviewer, Daron Acemoglu, Mehmet Barlo, Tarık Kara, Emin Karagözoğlu, Özgür Kıbrıs, Semih Koray, Yusufcan Masatlığlu, Eiichi Miyagawa, Kemal Yildız, as well as seminar participants at Bilkent University, Kadir Has University, ADA University, the Murat Sertel Workshop on Economic Design, the Conference on Economic Design, and the International Conference on Public Economic Theory. The financial support from the Japan Society for the Promotion of Science ("Topic-Setting Program to Advanced Cutting-Edge Humanities and Social Sciences Research"; KAKENHI 15H05729) is gratefully acknowledged. All remaining errors are our own.

\section{References}

Amir, R. (2005). Supermodularity and Complementarity in Economics: An Elementary Survey. Southern Economic Journal, 71(3), 636-660.

Aumann, R. (1959). Acceptable Points in a General Cooperative n-Person Games. in Contributions to the Theory of Games IV. Annals of Mathematical Study, 40, 287-324.

Berge, C. (1957). Théorie Générale des Jeux à n Personnes. Paris: Gautier Villars.

Bernheim, B. D., Peleg, B., \& Whinston, M. D. (1987). Coalition-proof Nash Equilibria I. Concepts. Journal of Economic Theory, 42(1), 1-12.

Bernheim, B. D., \& Whinston, M. D. (1987). Coalition-proof Nash Equilibria II. Applications. Journal of Economic Theory, 42(1), 13-29. 
Birkhoff, G. (1967). Lattice Theory. Rhode Island: AMS Colloquium Publications.

Calciano, F. L. (2007). Games with Complementarities. CORE Discussion Paper 16.

Calvo, E. \& Gutiérrez-López, E. (2015). The Value in Games with Restricted Cooperation. Working Paper, University of Valencia.

Carbonell-Nicolau, O., \& McLean, R. P. (2015). On Equilibrium Refinements in Supermodular Games. International Journal of Game Theory, 44, 869-890.

Chwe, M. S.-Y. (1994). Farsighted Coalitional Stability. Journal of Economic Theory, 63(2), 299-325.

Dutta, B., \& Sen, A. (1991). Implementation under Strong Equilibrium: A Complete Characterization. Journal of Mathematical Economics, 20(1), 49-67.

Echenique, F. (2005). A Short and Constructive Proof of Tarski's Fixed-point Theorem. International Journal of Game Theory, 33(2), 215-218.

Greenberg, J. (1989). Deriving Strong and Coalition-proof Nash Equilibria from an Abstract System. Journal of Economic Theory, 49(1), 195-202.

Karagözoğlu, E., Keskin, K., \& Sağlam, C. (2013). A Minimally Altruistic Refinement of Nash Equilibrium. Mathematical Social Sciences, 66(3), 422-430.

Keskin, K., \& Sağlam, C. (2014). Complementarities and the Existence of Strong Berge Equilibrium. RAIRO - Operations Research, 48(3), 373-379.

Keskin, K., Sağlam, C., \& Turan, A. (2014). General Complementarities on Complete Partial Orders. Fixed Point Theory and Applications, 2014(101).

Kimya, M. (2020). Equilibrium Coalitional Behavior. Theoretical Economics, 15(2), 669-714.

Kohlberg, E., \& Mertens, J.-F. (1986). On the Strategic Stability of Equilibria. Econometrica, 54(5), 1003-1037.

Konishi, H., Le Breton, M., \& Weber, S. (1997a). Equilibria in a Model with Partial Rivalry. Journal of Economic Theory, 72(1), 225-237.

Konishi, H., Le Breton, M., \& Weber, S. (1997b). Equivalence of Strong and Coalition-proof Nash Equilibria in Games without Spillovers. Economic Theory, 9(1), 97-113.

Konishi, H., Le Breton, M., \& Weber, S. (1999). On Coalition-proof Nash Equilibria in Common Agency Games. Journal of Economic Theory, 85(1), 122-139.

Maschler, M. (1963). $n$-person Games with only 1, $n-1$, and $n$-person Permissible Coalitions. Journal of Mathematical Analysis and Applications, 6(2), 230-256.

Milgrom, P., \& Roberts, J. (1996). Coalition-Proofness and Correlation with Arbitrary Communication Possibilities. Games and Economic Behavior, 17, 113-128.

Milgrom, P., \& Shannon, C. (1994). Monotone Comparative Statics. Econometrica, 62, 157-180.

Myerson, R. B. (1978). Refinements of the Nash Equilibrium Concept. International Journal of Game Theory, 7, 73-80.

Myerson, R. B. (1980). Conference Structures and Fair Allocation Rules. International Journal of Game Theory, 9(3), 169-182.

Nash, J. F. (1951). Non-cooperative Games. The Annals of Mathematics, 54, 286-295.

Pápai, S. (2004). Unique Stability in Simple Coalition Formation Games. Games and Economic Behavior, 48(2), 337-354.

Ray, D., \& Vohra, R. (1999). A Theory of Endogenous Coalition Structures. Games and Economic Behavior, 26(2), 286-336.

Selten, R. (1965). Spieltheoretische Behandlung eines Oligopolmodells mit Nachfrageträgheit - Teil I: Bestimmung des dynamischen Preisgleichgewichts. Zeitschrift für die gesamte Staatswissenschaft, $121,301-324$.

Selten, R. (1975). Reexamination of the Perfectness Concept for Equilibrium Points in Extensive Games. International Journal of Game Theory, 4, 25-55.

Topkis, D. M. (1998). Supermodularity and Complementarity. Princeton: Princeton University Press.

Vives, X. (2005). Complementarities and Games: New Developments. Journal of Economic Literature, $43,437-479$.

Xue, L. (2000). Negotiation-proof Nash Equilibrium. International Journal of Game Theory, 29(3), 339-357.

Zhou, L. (1994). The Set of Nash Equilibria of a Supermodular Game is a Complete Lattice. Games and Economic Behavior, 7, 295-300.

Publisher's Note Springer Nature remains neutral with regard to jurisdictional claims in published maps and institutional affiliations. 